\title{
Optimal Active Power Loss with Feeder Routing Collaborate Distributed Generation Allocation and Sizing in Smart Grid Distribution
}

\author{
Phatcharasak Phawanaphinyo ,Narongdech Keeratipranon, and Chaiyaporn Khemapatapan \\ College of Innovative Technology and Engineering, \\ Dhurakij Pundit University, Bangkok, 10210, Thailand \\ phatphaw@gmail.com
}

\begin{abstract}
The Smart Grid (SG) has widely supported the electric power from the Distributed Generation (DG). It becomes a practical standard to generate the electric power from a renewable energy into the distribution system to compensate for the power demand, especially in the peak time. However, the advancement of the SG continuing with the classical problem of active power loss as the traditional grid. This research aimsto solve the active power loss problem by analyzing the elements and study to solve, in the scope of the organization that provides the electrical power. In order to solve the problem, the solutions can be achieved by feeder routing with adjusting cost Dijkstra's algorithm, afterward decided the allocation and sizing of DG by using the Evolutionary Computing (EC) which are Harmony Search (HS), Artificial Bee Colony (ABC), and Particle Swarm Optimization (PSO) algorithms. The experiment evaluates the performance of the algorithm usingpower flow analysis, Backward/ Forward Sweep Method, on the IEEE 33-bus system. From the experimental result, the PSO provides the best performance. The overall active power loss in the case 3 DGs was reduced from 202.67 to $52.29 \mathrm{~kW}$, representing $74.20 \%$ reduction.
\end{abstract}

Keywords:Minimal active power loss, Backward \Forward Sweep Method, Harmony Search Algorithm, Artificial Bee Colony Algorithm, Particle Swarm Optimization Algorithm

\section{Introduction}

Asmart electricity system, such as a Smart Grid system, is required in order to better serve a higher power demand.The $\mathrm{SG}$ is an integration betweena traditional grid andInformation and Communication Technology (ICT) from the processes of Generation, Transmission, Distribution, to Consumption.In addition, from power plant electric sourcing, SG must be able to manage an extra power source from a Distributed Generation (DG) as well. Eventhough, the SG distribution has more advanced information but it still suffersthe same problem as the traditional grid, the active power loss, that directly affects the electrical power transfer. The problem above was a provocation, causing a significant money to improve the distribution line, distribution transformer, and substation building to accommodate the active power loss [1].

In related research, the defined radial distribution feeder routing with Dijkstra's algorithm to reduces the fixed cost, energy cost, and interruption cost [2].The other proposed the feeder routing for reducing the cost of energy loss by finding the shortest path to the distribution system [3]. In DG allocation and sizing, theHSwas used to solve the problem. The objective was determined by the voltage and the capacitor.In the experiments using the IEEE33-bus and IEEE 69-bus system to compared the results with the GAand Refine Genetic Algorithm(RGA).The results showed the HSwas the best efficient in processing time [4]. The PSOwas determine the allocation and sizing of the DG in the objective aimed to reduce the active powerloss. In the experiments with IEEE 33-bus system, the result reported the reduces active power loss by determining the allocation and sizing of the DG appropriately [5]. The ABChas chosen to experimental by modified the importance's parameters. In the objective function aimed to minimal the active power loss by experimented with IEEE 33-bus and IEEE69-bus system that installed the DG .The results modified ABC algorithm can reduce the processing time and number of iterations in the process as well [6].

Mostly the related research defined the objective function with minimal active power loss. Similarly, in this research is conduct toaforementioned objective function .In of order to solve the optimization 
problem, the experiment is select IEEE 33-bus system and process with feeder routing. After defined routing path, The DG allocation and sizing was experimental by compared the process ofHS, ABC and PSO.

\section{Proposed methodology}

\subsection{Adjusting Cost Dijkstra's Method}

The Dijkstra's algorithm had the ability to determining the shortest path from a source node to a destination. The shortest distance was calculated from the explicit costin the graph. On the other hand, the feeder routing is the variable, current and voltage, are change according to the direction of power flow. As a result, the feeder routing still having trouble to finding the exact value of the weight in the graph. In this paper has devised a feeder routing method with the Dijkstra's algorithm for determining the cost to solve the problem. The method has the ability to adjusting the cost, according to the direction of power flow. The cost is calculated from the power flow method. The calculation is repeated until the power variables are stable. The step of adjustingcost Dijkstra's methodwas composed of:

Step (1) Calculate routing cost. Define the initial values of $I_{K C L, i, t}$ equal $\infty$ and calculate the weight in the graph by the equation as:

$$
C_{i, t}=\tan ^{-1} \frac{I_{K V L, i, t}^{2} * X_{i}}{I_{K V L, i, t}^{2} * R_{i}}
$$

where $C_{i, t}$ is the cost of edges number $i$ that flow into node numberi in the time $t$, which calculate by thearctan of ratio between $I_{K V L, i, t}^{2} * X_{i}$ and $I_{K V L, i, t}^{2} * R_{i}$.

Step (2) Dynamic feeder routing process (Dijkstra's algorithm). The Dijkstra 's algorithm was modified by separatedfor routing as the process to:

(2.1) Find shortest with Dijkstra 's algorithm, the process of finding the shortest path from the Dijkstra's algorithm from one source to all destinations.

(2.2) Cut the duplicate edges, by cut the overlapping edges.

(2.3) Eliminate multi-source node, by selecting the node with the lowest total cost to the source node and cut others off.

(2.4) Move the end node to compare active power loss, the results can be separated into two cases. If moving the node and then active power loss reduce, (2.4.1) move the end node to the minimal active power loss source node. Then, recursive to process (2.3) until can 't minimal active power loss, the result is (2.4.2) Adjusting Cost Dijkstra's path.

Step (3) Calculate with power flow analysis. The power flow parameters are calculating the new current in time period $t+1$, in the term of $I_{K C L, i, t+1}$ for the purpose of adjusting the new cost in the term of $C_{i, t+1}$.

Step (4) Find the tolerant between $\boldsymbol{C}_{\boldsymbol{i}, t+1}$ and $\boldsymbol{C}_{\boldsymbol{i}, \boldsymbol{t}}$. In the comparison, if tolerant is more than or equal 0.0001 (4.1), the cost isadjustedby assigned with $C_{i, t+1}$, and repeat from the second step . On the contrary, if the tolerant is less than 0.0001 (4.2), the result will be the lower active power losspath with the right cost. In the process update the new cost, the improvement will replace the higher cost.Thus, according to the Dijkstra 's algorithm, that routing to the lowest cost, the edges will be choose and improved the cost repletely until steady. Finally, the shortest path was defined with the actual cost.

\subsection{Problem Formulation}

Since the active power loss is depended on the current injected through the branch, that calculated with Ohm's Law. Moreover, the installation of DG effect to change the activepower and the current. Accordingly, in this research is definedthe objective function in the active power loss as follows:

$$
\text { Minimal } \sum_{i=1}^{n} I_{K C L, i}^{2} R_{i},
$$

where the $I_{K C L, i}$ is current (Ampere) of the branch that inject into bus number $i$, which can be calculated by the power flow method and $R_{i}$ was the resistance of branch that flows into the bus 
number $i$. The Constraints in experimentare included with the parameters of bus and branch that compose of current, voltage, active power, reactive power and appearance power.

$$
\begin{gathered}
I_{K C L, i} \leq I_{\text {imax }}, \\
V_{\text {imin }} \leq V_{K V L, i} \leq V_{\text {imax }},
\end{gathered}
$$

where $I_{K C L, i}$ is the current (Ampere) that limit to not more than $I_{M A X, i}$, in the condition of the maximum current flow in conductor type in branch $i$. The voltage level of the bus must not be higher or lower voltage limit, the lower and upper limits of voltagesecurity range are set to be 0.90 p.u. and 1.05 p.u. [7]. In the term of $V_{K V L, i}$ is voltage (Volt) in bus number $i$ that the value is not less than $V_{\text {imin }}$ and not more than $V_{\text {imax }}$. In terms of active power and reactive power produced from DG must be limit of the bus. The constraints that shown as:

$$
\begin{aligned}
& P_{D G, \text { imm }} P_{D G, i} \leq P_{D G, \text { imax }} \\
& Q_{D G, \text { im市 }} Q_{D G, i} \leq Q_{D G, \text { imax }}
\end{aligned}
$$

where $P_{D G, i}$ is the active power (Watt) in bus numberi that supply power in the range of $P_{D G, i m i n d}$ $P_{D G, i m a}$ Similarly, $Q_{D G, i}$ is the reactive power (VAR) in bus number $i$ that supply power in the range of $Q_{D G \text {, imind }} Q_{D G, \text { imd }}$ Xoreover, the appearance power of DG must not exceed the power requirement in all bus. That can show as:

$$
\sum_{i=1}^{i} \sqrt{P_{D G, i}^{2}+Q_{D G, i}^{2}} \leq \sum_{i=1}^{i} \sqrt{P_{\text {Load }, i}^{2}+Q_{\text {Load }, i}^{2}},
$$

where the equation (7) represents the all appearance power from that generated from DG must not more than all appearance power on the bus.

\section{Result and Discussion}

The experimental use of data and topology of IEEE 33-bus system [8]. The nodes were composed of the slack bus and load bus are represented by a square and a circle, respectively. In terms of the branch is represented by the directed edges. Graph topology is a directed graph which direction efficacy the power flow by edges. The important variables arecomposed of static variables in each node consisting of Active Power $\left(P_{i}\right)$, the unit as Watt, Reactive Power $\left(Q_{i}\right)$, the unit as VAR. The variables of edges are contained with Resistance $\left(R_{i}\right)$ and Reactance $\left(X_{i}\right)$ by the couple a unit as Ohm.

The experimentationthat aimed to minimal the active power loss was divided into two section. The first was the feeder routing with adjusting cost Dijkstra methodand the second, in the consequent process, the DG allocation and sizing with EC. The EC methods, HS, ABC and PSO, were compared. The standard model, IEEE 33-bus system, was chosen and defined the voltage base was $12.66 \mathrm{kV}$ and the apparent power base was $10 \mathrm{MVA}$, which shown results into the following topics.

\subsection{Feeder routing with Adjusting Cost Dijkstra's method.}

The accuracy in the calculation of active power loss can be verified with related research that routing on base path. open the tie-switches between the nodes 8-21, 9-15, 12-22, 18-33, and 25-29. All the active power and reactive power wereequal $3,715.00 \mathrm{~kW}$ and $2,300.00 \mathrm{kVAR}$, respectively. In the test of routing with base path in this work the power loss was $202.66 \mathrm{~kW}$, that accurate as the other works [17], [9], [20].After verified the accuracy, next step was adjusting cost Dijkstra method. The feeder routing can be routed to the lower active power path with adjusted $C_{i, t}$ in 4 iterations. From the Dynamic Dijkstra's path was changed topology by the open the switch between node 7-8, 9-10, 14-15, 25-29 and 32-33. The results showed the decision of adjusting cost Dijkstra's had reduced active power loss to $139.56 \mathrm{~kW}$.

\subsection{DG allocation and sizing}

In the DG allocation and sizing was compared between HS, ABC, and PSOfrom the Dynamic Dijkstra's path. The DGswere penetrated in thegraph as P-Q bus. The nodes of graph on the IEEE 33-bus system were defined as the problem dimensions. The analysis consists of 4 case, accordance 
with the maximum supply not over than active load, Case-I: 1 DG with the maximum supply 1,000.00 $\mathrm{kW}$, Case-II: 1 DG with the maximum supply 2,000.00 kW, Case-III: 2 DGs with the maximum supply1,000.00 kW, and Case-IV: 3 DGsthe maximum supply1,000.00 kW. In the first stage using Case-I and Case-II to compare the results to find the appropriate method, between HS, ABC, and PSO. The parameters of PSO algorithm were composed ofthe problem dimensions was 32, the weight was 0.3 , the swarm size was 100 , andthe learning factors $a_{2}$ and $a_{2}$ are 1.5 . The parameters of the $\mathrm{ABC}$ algorithm were determined by the best result, that consists of nectar source were 32 , scout bees were 70, employed bee was 35 and onlooker bees was 35. Similarly, in the parameters of HS algorithm were composed of: Vector of Harmony Size (HMS) was32,Harmony Memory (HM) was30, Harmony Consideration Rate (HCR) was0.9, Pitch Adjust Rate (PAR) was0.3, and Bandwidth $(b w)=$ 0.01. All methods determination optima's iterations, the processing by iteration until the best cost from objective function don't change more than the tolerance that equal 0.0001 in the 100 -previous rounds. The experimentation was 10 times for compared the power loss (best cost), node allocation, DG sizing, optima's iterations, and optima' processing time. The results werecomparedin the term of mean and standard deviation $(\sigma)$ values, which shown Table 1.

Table 1. Compare result betweenHS,ABC and PSO.

\begin{tabular}{|c|c|c|c|c|c|c|}
\hline \multirow{3}{*}{ Indicators } & \multicolumn{6}{|c|}{ Algorithms } \\
\hline & \multicolumn{2}{|c|}{ HS } & \multicolumn{2}{|c|}{$\mathbf{A B C}$} & \multicolumn{2}{|c|}{ PSO } \\
\hline & Mean & $\sigma$ & Mean & $\boldsymbol{\sigma}$ & Mean & $\boldsymbol{\sigma}$ \\
\hline \multicolumn{7}{|c|}{ Case-I: DG allocation node [30] } \\
\hline Power loss $(\mathrm{kW})$ & 93.15 & 0.4443 & 92.68 & 0.0000 & 92.68 & 0.0000 \\
\hline DG sizing $(\mathrm{kW})$ & 985.35 & 13.1325 & 1000.00 & 0.0000 & 1000.00 & 0.0000 \\
\hline Optima's iteration(times) & 82.33 & 14.3233 & 3.56 & 1.4181 & 2.00 & 0.0000 \\
\hline $\begin{array}{l}\text { Optima's processing time } \\
\text { (seconds) }\end{array}$ & 7.08 & 1.2501 & 11.84 & 4.5420 & 1.59 & 0.0564 \\
\hline \multicolumn{7}{|c|}{ Case-II: DG allocation node [25] } \\
\hline Active power loss $(\mathrm{kW})$ & 85.41 & 0.1835 & 84.97 & 0.0000 & 84.97 & 0.0000 \\
\hline DG sizing $(\mathrm{kW})$ & $1,911.07$ & 51.0407 & 1762.36 & 0.0000 & 1762.36 & 0.0000 \\
\hline Optima's iteration (times) & 47.44 & 26.6352 & 82.67 & 17.9010 & 42.33 & 8.6127 \\
\hline $\begin{array}{l}\text { Optima's processing time } \\
\text { (seconds) }\end{array}$ & 4.06 & 2.2555 & 318.75 & 69.9454 & 42.00 & 7.7876 \\
\hline
\end{tabular}

In the Case-I, all methods were install DG on node 30. The HS defined the mean active power nearly optimal capacity $(1,000.00 \mathrm{~kW})$, while ABC and PSO can be implemented to themaximum DG capacity. In the terms of reduce active power loss, the ABC and PSO can reduce active power loss equal $92.68 \mathrm{~kW}(\sigma=0.0000)$. The HS was found the mean active power loss on $93.15 \mathrm{~kW}(\sigma=0$. 4443). In the optima's iteration and processing time, HS was showed very high, mean on 82.33 times $(\sigma=14.3233)$ and $7.08(\sigma=1.2501)$, respectively. The results of the ABC and PSO algorithm was similarly. The active power loss in both algorithms were optimal in the best cost at $92.68 \mathrm{~kW}$ $(\sigma=0.0000)$ and the DG sizing were presented at $1,000.00 \mathrm{~kW}(\sigma=0.0000)$.

In the Case-II, all methods were install DG on node25. The DG sizing was not the maximum capacity for reason to made the voltage profile at the node to meted the minimum active power loss. The DG sizing in the HS means at $1,911.07 \mathrm{~kW}(\sigma=51.0407)$, while the ABC and PSO couldachieve in $1,762.36 \mathrm{~kW}(\sigma=0.0000)$. In terms ofactive power loss reduction, the ABC and PSO can get better by in the both cases equal $84.97 \mathrm{~kW}(\sigma=0.0000)$. In the optima's iteration and the processing time, the PSO algorithm was the best performance at 42.00 seconds $(\sigma=7.7876)$.

In contrast, the ABC and PSO had given the better results in the processing, especially PSO. In the first section the PSO algorithm was the best performance. In the second section,the consequent 
experiments,according to Case-III and Case-IV has selected PSO. The empirical model based on the previous parameter values of tolerance and the number of iterations required. The experimented was conducted in 10 times, which can show results as Table 2.

Table 2. The result of experimented with PSO in Case-III and Case-IV.

\begin{tabular}{|l|r|r|r|r|}
\hline & \multicolumn{2}{|c|}{ Case-III: DG allocation nodes [8 30] } & \multicolumn{2}{c|}{ Case-IV: DG allocation nodes [8 24 31] } \\
\hline \multicolumn{1}{|c|}{ Performanceindicators } & \multicolumn{2}{|c|}{ Mean } & \multicolumn{1}{c|}{ Mean } & \multicolumn{1}{c|}{$\boldsymbol{\sigma}$} \\
\hline Active power loss (kW) & 70.56 & 0.0000 & 52.29 & 0.0000 \\
\hline DG sizing (kW) & {$[920.741000 .00]$} & - & {$[899.42994 .48962 .50]$} & - \\
\hline Optima's iteration (times) & 45.56 & 7.3492 & 69.78 & 71.79 \\
\hline Optima's time (seconds) & 48.34 & 9.9009 & 52.29 & 10.4350 \\
\hline Active power loss (kW) & 70.56 & 0.0000 & 0.3602 \\
\hline
\end{tabular}

In the Case-III, the DG was installed in the node 8 and 30,with the total sizing of 920.74 and $1,000.00 \mathrm{~kW}$. The mean active power losswas $70.56 \mathrm{~kW}(\sigma=0.0000)$. In terms of the processing performance, the number optima'siterations and processing time to were 45.56 times $(\sigma=7.3492)$ and 48.34 seconds $(\sigma=9.9009)$, that showed in Fig. 1.

The final case, Case-IV, the results showed the DG allocation will be installed in the node 8, 24 and 31 with the sizing were $899.42,994.48$, and $962.50 \mathrm{~kW}$. The activepower lossreduced to $52.29 \mathrm{~kW}$ $(\sigma=0.0000)$. In terms of efficiency of processing, was stableinferior to 1 DG. In the optima's iteration and processing time, were 69.78 times $(\sigma=10.4350)$ and 71.79 seconds $(\sigma=10.3602)$, respectively.
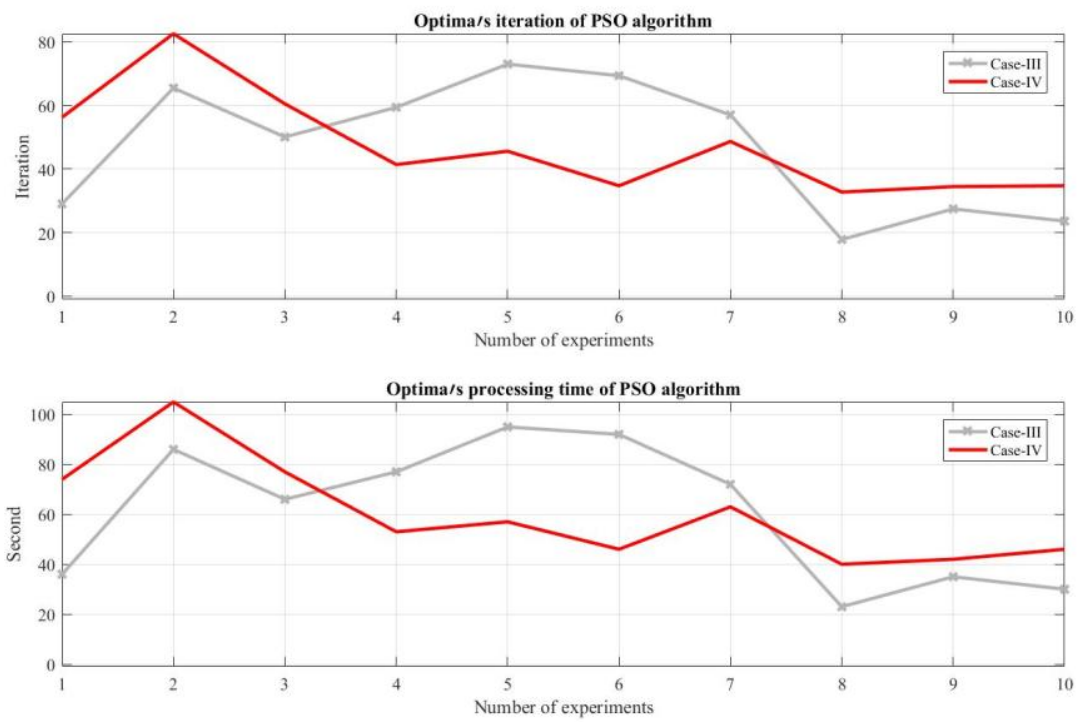

Figure 1.The Optima's iteration and processing time with PSO.

In comparison, the results in the Case-III and Case-IV, was defined allocation and sizing were determined exactly. In contrast, the values of optima'siterations and processing time were not stable due to the $\sigma$ value. In addition of defining the objective function to minimal the active power loss, can be achieved effectively. In the best case, the process of feeder routing and optimization the DG allocation and sizing in Case-IV, the active power loss was reduced from 202.67 to $52.29 \mathrm{~kW}$, representing $74.20 \%$. The steps of minimal active power loss from the feeder routing and reduced to $139.56 \mathrm{~kW}$ and the consequence with DG allocation and sizing by PSO was reduce to $52.29 \mathrm{~kW}$.

\section{Conclusions}

The research aimed to analyzed the active power loss problems and found the variety of possible solutions. The solution of feeder routing with adjusting cost Dijkstra' method, consequently DG allocation and sizing with PSO, can reduce active power loss as well. The approach of the feeder 
routing with Dijkstra's algorithm can be applied to adjusted the cost in the concept of dynamic for the match the real power flow. After adjusted the cost, the experimentation was reconfiguration feeder topology, that implemented effectively. Consequently, the DG allocation and sizing were the experiment by the EC for reducing active power loss. The experimented found that PSO algorithm could determine the proper location and sizing of DG. Both methods have solved the active power loss problem in a coherent system. However, this research work can be further studied to cover a multi-objective function by adding Power Factor for a more effective measurement. Furthermore, the experiment data will adjust to time series, with historical load and supply from the real feeder.

\section{References}

[1]Xiang-min, H., Yong-jun, Z., and Huan-cai, H. 2014. Automatic Reactive Power Control in Distribution Network Based on Feeder Power Factor Assessment, IEEE Region 10 Symposium, Indonesia, 1-4.

[2]Priya, J., and Vidyasagar, S. 2013. Dijkstra Algorithm for Feeder Routing of Radial Distribution System, IOSR Journal of Engineering, January 20131-6.

[3]Jha, P., \&Vidyasagar, S. (2013). Dijkstra Algorithm for Feeder Routing of Radial Distribution System. IOSR Journal of Engineering, Volume 3, Issue 1, January 2013, pp.1-6.

[4]Rao, R.S., Ravindra, K.,Satish, K., and Narasimham, S.V.L. 2013. Power Loss Minimization in Distribution SystemUsing Network Reconfiguration in thePresence of Distributed Generation. IEEE Transactions on Power Systems,28,317-325.

[5]Guerriche, K.R. and Boukti, T. 2015. Optimal Allocation and Sizing of Distributed Generation with Particle Swarm. Revue des Sciences et de la Technologie(RST),6, 59-69.

[6]Abu-Mouti, F.S., and El-Hawary, M.E. 2009. Modified Artificial Bee Colony Algorithm for Optimal Distributed Generation Sizing and Allocation in Distribution Systems. Electrical Power \& Energy Conference (EPEC), 2009, 1-9.

[7] Alafnan, H., Zhao, J., and Ma, W. (2016). Prevention of overvoltage induced by large penetration of photovoltaics in distribution networks by electric vehicles. IEEE Transportation Electrification Conference and Expo, Asia-Pacific (ITEC Asia-Pacific), 2016, 5253-530.

[8]Baran, M.E., \& Wu, F.F. (1989). Network Reconfiguration in Distribution Systems for Loss Reduction and Load Balancing. IEEE Transactions on Power Delivery, Vol. 4, No. 2, April 1989, pp.1401-1407. 